
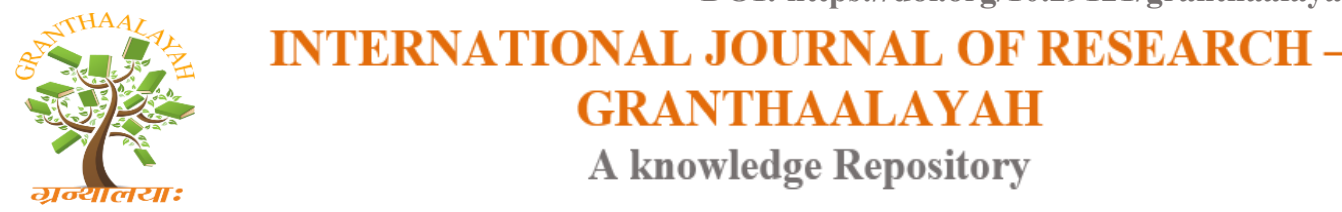

Science

\title{
A REVIEW PAPER ON STRUCTURAL DAMAGE IDENTIFICATION OF CANTILEVER BEAM BY USING VIBRATION TECHNIQUE, FINITE ELEMENT ANALYSIS \& ARTIFICIAL NEURAL NETWORK
}

\author{
Aakanksha Mohanrao Garud *1, Prof. V. G. Bhamre *2 \\ ${ }^{*}$ PG Scholar, Department of Mechanical Engineering, SNDCOE \& RC, Yeola, S. P. Pune \\ University, INDIA \\ ${ }^{* 2}$ Head, Department of Mechanical Engineering, SNDCOE \& RC, Yeola, S. P. Pune University, \\ INDIA
}

\begin{abstract}
In this review paper structural damage identification work in cantilever beam is done by using the Artificial Neural Network as diagnostic parameter. The study is based on the concept that natural frequency is inversely proportional to the mass of the structure. Thus to regulate the proper condition of structure, periodical frequency measurement is necessary. But in dynamic conditions and in complicated structures frequency measurement is difficult, for the same we reviewed various papers to identify the structural damage using various methods. The factors which affects on the damage of structural parts like crack depth, crack location etc. is also discussed in this work. Natural frequency is measured with the help of fast fourier transform by various authors and artificial neural network is also used for identification of the damage in many papers. So in this review work we studied methods of structural damage identification such as vibrations, finite element analysis and artificial neural network.
\end{abstract}

Keywords:

damage identification, artificial neural network, natural frequency, condition of structure, finite element analysis, fast fourier transform.

Cite This Article: Aakanksha Mohanrao Garud, and Prof. V. G. Bhamre, "A REVIEW PAPER ON STRUCTURAL DAMAGE IDENTIFICATION OF CANTILEVER BEAM BY USING VIBRATION TECHNIQUE, FINITE ELEMENT ANALYSIS \& ARTIFICIAL NEURAL NETWORK" International Journal of Research - Granthaalayah, Vol. 3, No. 12(2015): 125-128.

\section{INTRODUCTION}

Presence of structural damage in a mechanical part changes the modes of vibrations. These changes in measuring parameters are shown in the modes of vibration which can be obtained with the help of finite element analysis. In the literature review generally the terms natural frequency, modal analysis, modes, crack depth, crack location are used. All this refers to the 
damage identification vulnerable parameters. But for every mode the frequencies are different for different damage location and depth. To identify damage, the results are used from dynamic testing by various authors. As time changes the results are same in some cases and sometimes it is coinciding the periodical inspection, also another advantage is to relate the natural frequency with the cracks.

Marjan Djidrov et al (2014) worked on "Vibration Analysis of Cantilever Beam for Damage Detection", in this paper; by using finite element analysis software vibration analysis and frequency response analysis of cantilever aluminum beam with bonded piezoelectric transducer were presented. By location and depth of single transversal crack, vibration response of cantilever beam was analyzed and numerical results of undamaged beam model are compared with damage present in structure. Effect of different variables on beam model, changes in first three natural frequency and FRF amplitude were also presented. Results obtained by ANSYS software of Vibration behavior of the beam shows that cantilever beam model was sensitive to the variables like crack location, crack depth and vibration modes. Results shows that near the fixed end highest variation of natural frequencies occurs for the first mode of beam vibration at crack position ${ }^{[1]}$. Kaustubha V. Bhinge et al (2014) worked on "Crack Detection in Cantilever Beam by Vibration Techniques". Their study was related to measurement of natural frequency, a global parameter that can be easily measured at any point conveniently on the structure. To give the relationship between the stiffness \& location of crack it is simulated by a spring in theoretical analysis, connecting the two segments of the beam. Using finite element method the model of beam is generated ${ }^{[2]}$. Dr. P. K. Sharma et al (2014) worked on "Alternative Solution to the Detection of Crack Location and Crack Depth in Structure by Using Software Analysis Method". Here in this study they used ANSYS software package for finite element analysis of crack and un-crack cantilever beam taking input file as a CAD design developed in CATIA. Experiments was done over 10 models of crack beam which were having different cross section, Changes in some of the lower natural frequencies were because of the presence of crack. The proposed methodology has been verified by comparing results obtained from theoretical method and finite element analysis. This study concludes that the natural frequency of the beam decreases when the crack depth increases and the crack location is constant. At the area of crack location Natural frequency changed ${ }^{[3]}$. Akhilesh Kumar et al (2014) worked on "Experimental investigation of crack in aluminum cantilever beam using vibration monitoring technique". In this paper an experimental setup was designed in which by using power exciter and accelerometer an aluminum cantilever beam with cracks is excited. To avoid non-linearity the cracks are assumed to be open. The experimental investigation gives effects of crack and positions on the fundamental frequencies of slender cantilever beams with edge cracks. The experiments were conducted using specimens having different variables at different positions to validate the numerical results obtained. From digital storage oscilloscope (DSO) the experimental results of frequencies were obtained. The basic criterion for crack detection was first three natural frequencies. From the results it can be observed that the vibration behavior of the beams was very sensitive to the crack location, crack depth and mode number ${ }^{[4]}$. Prasad R. Baviskar et al (2014) worked on "Experimental verification of detection and prediction of multiple cracks by vibrations, FEM and ANN", a method for recognition and divination of properties of multiple transverse cracks on simply supported stepped rotor shaft were studied by author. They considered two cases of cracks. Firstly, both cracks were at right angle to axis. Later, those cracks were inclined at an angle with vertical plane and also inclined with each other. To get 
natural frequency and mode shapes modal analysis was carried out. Finite element method was considered as basis for numerical analysis. Using fast fourier transform analyzer validation and experimentation was carried out. Cracks were identified based on natural frequency. By using artificial neural networks crack properties were identified in forward technique. To train the network of ANN to predict the crack properties database of natural frequencies were used. Applicability of the method was validated by comparing the divinations of ANN with FEM and experimentation. The divinations of ANN and results came out from FEM and experimentation were found in agreement ${ }^{[5]}$. A. Esfandiari et al (2013) worked on "Theoretical and experimental structural damage diagnosis method using natural frequencies through an improved sensitivity equation", a frequency-based technique was presented by authors to diagnose damages induced stiffness reduction in a structure. A second order element level function of the stiffness reduction was distinguished by the sensitivity of natural frequencies. To achieve precise results, a linear combination of the intact structure mode shapes were expressed by change in the mode shapes of the structure, and were taken in to account to develop sensitivity equations. An indeterminate set of equations were yielded by overall formulation. To solve these equations an optimization criterion was used to obtain the change of the structural parameters. Results show the efficiency of the proposed method and the importance of including the changes of the mode shapes through a frequency-based damage detection algorithm and also approve that the method was able to detect damage without an exact model of the undamaged structure ${ }^{[6]}$. Kaushar H. Barad et al (2013) worked on "Crack detection in cantilever beam by frequency based method", using natural frequency recognition of the damage exist on the surface of structural element was explained by authors. For identification of damage parameters first two natural frequencies of the cracked beam were obtained experimentally and used. Detected crack locations and size were compared with the actual results and found to be in good agreement. Also on the damage parameters the effect of the natural frequency was discussed. Using this approach, damage detection can be done with the help of natural frequency. The natural frequency was greatly affected by crack depth and crack location. From result it was observed that the crack of a particular size present near the fixed end minimizes the natural frequency significantly greater than the crack of that size present closer to the free end. It was also noticed that the higher crack to depth ratio has great effect on the normalized natural frequency than that of smaller ratio.

\section{CONCLUSION}

It can be conclude that cantilever beam model is sensitive to the crack location, crack depth and vibration modes. The crack depth and natural frequency are inversely proportional to each other while the crack location is kept constant. While the crack depth keeping constant natural frequency decreases with increase in crack location from the cantilever end. As cross section of square beam have same cross and longitudinal frequency, it is suitable to take in to consideration rectangular cross section beams since they have larger transverse frequency than longitudinal. It is obvious that the vibration behavior of the beams is very sensitive to the crack location, crack depth and mode number. The direction of crack does not affect the natural frequency. Crack with larger crack to depth ratio $(\mathrm{a} / \mathrm{h})$ imparts greater reductions in natural frequency than that of the smaller. 


\section{ACKNOWLEDGEMENT}

I would like to express my sincere thanks and appreciation towards Prof B. C. Londhe and Prof. A. P. Ghodake for their continuous guidance and valuable support in this work.

\section{REFERENCES}

[1] Marjan Djidrov et al, Vibration Analysis of Cantilever Beam for Damage Detection, FME Transactions, Volume 42, No. 4, 2014, 311-316.

[2] Kaustubha V. Bhinge et al, Crack Detection in Cantilever Beam by Vibration Techniques, IJAERS, July 2014, 80-86.

[3] Dr. P.K. Sharma et al, Alternative Solution to the Detection of Crack Location and Crack Depth in Structure by Using Software Analysis Method, IJARSE, Volume 3, Issue 8, August 2014, 181-186.

[4] Akhilesh Kumar, Experimental investigation of crack in aluminum cantilever beam using Vibration monitoring technique, IJCERC, Volume 4, Issue 4, April 2014, 39-50.

[5] Prasad Ramchandra Baviskar, Experimental verification of detection and prediction of Multiple Cracks by Vibrations, MMMS, Volume 10, No. 3, 2014, 290-303.

[6] A. Esfandiari, Theoretical and experimental structural damage diagnosis method using natural frequencies through an improved sensitivity equation, IJMS, 2013, 79-89.

[7] Kaushar H. Barad, Crack detection in cantilever beam by frequency based method, ELSEVIER, 2013, 770- 775. 